\title{
Selection of the optimal number of shifts in fuzzy environment: manufacturing company's facility application
}

\author{
Sanjoy Kumar Paul, Abdullahil Azeem \\ Bangladesh University of Engineering and Technology (BANGLADESH) \\ sanjoy@ipe.buet.ac.bd; azeem@ipe.buet.ac.bd
}

Received May 2009

Accepted March 2010

\begin{abstract}
This paper addresses the selection of optimal shift numbers considering inventory information, customer requirements and machine reliability using fuzzy logic. Number of shift is one of the most important criteria for the production planners to minimize the production costs and is essential for appropriate production planning. The main task involves optimizing the shift periods considering constraints of raw material, due date, demand, finished goods inventory and machine breakdown. A model is developed for any kind of manufacturing company where shift periods affect company's profit and cost. Fuzzy control is used to optimize the number of shifts under the constraints of raw material, due date, demand, finished goods inventory and machine breakdown. MATLAB Fuzzy Logic Tool Box is used to develop the model.
\end{abstract}

Keywords: optimal shift period, fuzzy logic, fuzzy set, production planning

\section{Introduction}

In this competitive age of manufacturing, factories are being forced to use multiple shift production to meet customers' requirements. However, determining optimal number of shift is very important as it involves huge cost that affects company's profit. Direct labor cost is the prime factor which is directly related to number of shifts. Besides, there are factory overhead costs as well as general and administrative costs that are also proportional to number of shifts. As all these 
costs are directly proportional to the number of shifts, reducing this objective function (number of shifts) can significantly decrease the operational costs. Consideration of constraints is one of the important tasks to develop the model because constraints may differ depending on the type of company. After selecting the appropriate constraints of raw material, due date, demand, finished goods inventory and machine breakdown a model is developed using fuzzy logic. Numerous studies have been conducted in the area of fuzzy environment. Graves (1985) presented a model of a multi-echelon spare parts inventory system where the response time to a failure consists of the transit time to deliver the failed part from the depot to the warehouse, the time spent waiting for repair at the warehouse, the repair time at the warehouse, and the transit time to return the repaired part from the warehouse back to the depot. Axsater (1990) presented an inventory system that consists of one warehouse and multiple depots with constant warehouse replenishment lead times under a base stock policy and constant transportation times from the warehouse to the depots. Caglar (2001) also presented a multi-echelon spare parts inventory model with time response constraints. Hopp, Zhang and Spearman (1999) presented a two-echelon spare parts inventory optimization model that minimizes total inventory investment subject to a maximum allowable total delay per facility. Svornos and Zipkin (1991) presented one of the first studies to address probabilistic transit time. They consider a multi-echelon inventory system utilizing a base stock inventory policy. Cohen, Kleindorfer, Lee and Lee (1986) develop a multi-echelon inventory model with probabilistic time response constraints. In this model, the demand resulting from part failures in the field is supported by stock held at stocking points situated at various levels in the system. Petroni and Rizzi (2002) used fuzzy logic based methodology to rank shop floor dispatching rules. Grabot and Geneste (1994) proposed a way to use fuzzy logic in order to build aggregated rules allowing obtaining a compromise between the satisfactions of several criteria. McCahonE and Lee (1992) used fuzzy logic for job scheduling in a flow shop. Allet (2003) dealt with a particular scheduling problem inspired by a practical case coming from a Belgian Pharmaceutical company. Balan, Vrat and Kumar (2007) used fuzzy logic and showed that the Bullwhip effect can be reduced by replacing the P-controller with a Fuzzy Logic Controller (FLC). The energy regenerative braking system (ERBS) of the CFA6470 parallel hybrid electric vehicle (CFA6470PHEV) was designed using the 
T-S fuzzy model and fuzzy control toolbox in the MATLAB by Deng, Gong, Yang, and Tan (2008). Serra and Bottura (2008) dealt with the development of a robust fuzzy observer in the presence of disturbances. In this approach, the chosen instrumental variables, statistically uncorrelated with noise, are mapped to fuzzy sets, partitioning the input space in subregions to define unbiased estimates of the TS fuzzy model consequent parameters in a noisy environment. A realistic inventory model with imprecise inventory costs formulated for deteriorating items under inflation by Mahata and Goswami (2009). Hedjar (2008) dealt with the design of a fuzzy controller for a production inventory system with deteriorating items, where the deterioration rate is unknown and the demand rate depends on the inventory level in a non-linear relationship.

No work has been found so far to find out optimal number of shifts for a manufacturing company. This paper focuses on calculating optimal number of shifts using fuzzy logic having constraints of raw material, due date, demand, finished goods inventory and machine breakdown.

\section{Modeling of production system}

Fuzzy logic is an optimization technique which considers different inputs and relates those inputs with output with some rules. Rules indicate the relationship between inputs and outputs. The output is optimized based on relationship between variables. The final output is obtained from the aggregated optimized result of individual rule.

Fuzzy set theory was originally presented by Zadeh (1965) in his seminal paper "Fuzzy Sets" in Information and Control. However, some of the key ideas were already envisioned by Black in 1937. Fuzzy logic was developed later from fuzzy set theory primary to reason with uncertain and vague information and secondary to represent knowledge in operationally powerful form (Frantti \& Mähönen, 2001).

Fuzzy logic is a method to formalize the human capacity of imprecise or approximate reasoning. Such reasoning represents the human ability to reason approximately and judge under uncertainty. In fuzzy logic all truths are partial or approximate. In this sense this reasoning has also been termed interpolative reasoning, where the process of interpolating between the binary extremes of true and false is represented by the ability of fuzzy logic to encapsulate partial truths 
(Ross, 1995). Five main steps are involved in modeling a fuzzy expert system which is shown in Figure 1 (Ordoobadi \& Mulvaney, 2001):

- Define the input variables for the system and their corresponding ranges of values.

- Define the output variables or the system and their corresponding ranges of values.

- Develop fuzzy membership function for every input and output.

- Develop a rule base based upon the potential outcomes of the system.

- Determine how each action will be carried out by establishing the rule strengths and defuzzification. The input for the defuzzification process is a fuzzy set (the aggregate output fuzzy set) and the output is a single number. As much as fuzziness helps the rule evaluation during the intermediate steps, the final desired output for each variable is generally a single number. However, the aggregate of a fuzzy set encompasses a range of output values, and so must be defuzzified in order to resolve a single output value from the set. Perhaps the most popular defuzzification method is the centroid calculation, which returns the center of area under the curve. There are five built-in methods supported: centroid, bisector, middle of maximum (the average of the maximum value of the output set), largest of maximum, and smallest of maximum.

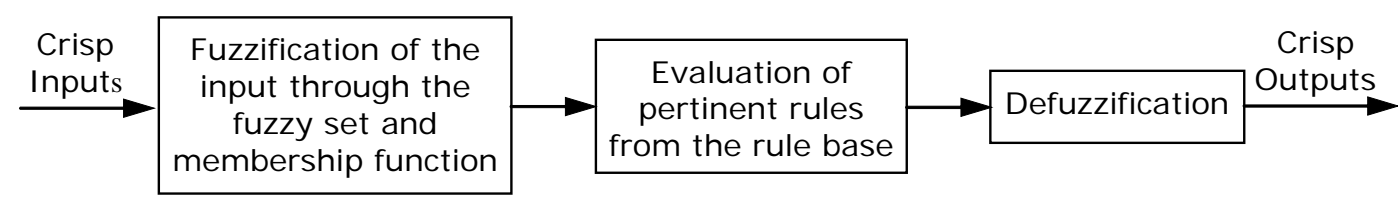

Figure 1. "General depiction of Fuzzy Expert System". Source: Own contribution

If general depiction of fuzzy expert system is adopted in Figure 1, the selecting of optimal shift numbers' fuzzy model can be presented in Figure 2. 


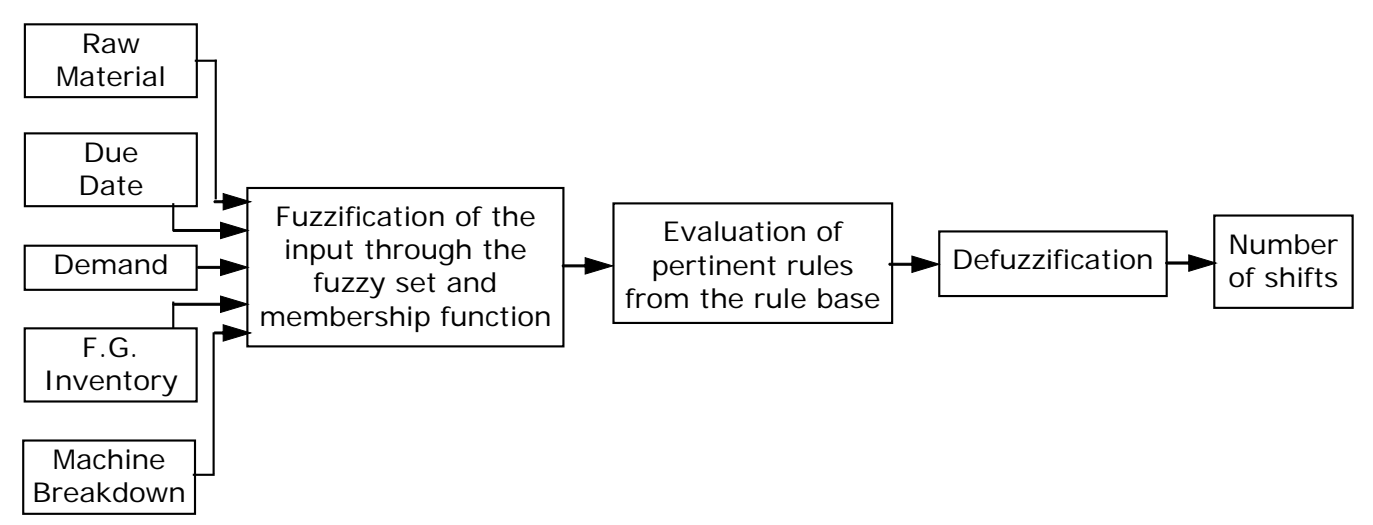

Figure 2. "Fuzzy Model of proposed system". Source: Own contribution

Novák, Perfilieva and Mockor (1999) discussed the mathematical principles of fuzzy logic in their book. According to this, mathematical principles of Fuzzy Logic provides a systematic study of the formal theory of fuzzy logic. This book presents fuzzy logic as the mathematical theory of vagueness as well as the theory of commonsense human reasoning, based on the use of natural language, the distinguishing feature of which is the vagueness of its semantics. Gottwald (2005) developed the mathematical formulation of fuzzy logic, known as mathematical fuzzy, which is considered as an approximate reasoning technique of fuzzy logic.

\begin{tabular}{|l|c|c|c|}
\hline \multicolumn{1}{|c|}{ Input variable } & Low/ Short & Medium & High/ Long \\
\hline Raw Material (pieces) & $100-400$ & $300-600$ & $550-900$ \\
\hline Due Date (days) & $1-10$ & $8-30$ & $25-50$ \\
\hline Demand (units) & $0-150$ & $150-300$ & $300-600$ \\
\hline $\begin{array}{l}\text { Finished Goods } \\
\text { Inventory(units) }\end{array}$ & $0-100$ & $100-300$ & $300-600$ \\
\hline $\begin{array}{l}\text { Machine Breakdown } \\
\text { (Times per day) }\end{array}$ & $0-1$ & $1-3$ & $3-5$ \\
\hline
\end{tabular}

Table 1. "Variation of input linguistic variables". Source: Own contribution

\begin{tabular}{|c|c|c|c|c|c|}
\hline $\begin{array}{c}\text { Output } \\
\text { Variable }\end{array}$ & Very Low & Low & Medium & High & Very High \\
\hline $\begin{array}{c}\text { Number of } \\
\text { Shift per } \\
\text { day }\end{array}$ & $0-0.5$ & $0.5-1$ & $1-2$ & $2-2.5$ & $2.5-3$ \\
\hline
\end{tabular}

Table 2. "Variation of output linguistic variables". Source: Own contribution

While generating the fuzzy model's linguistic variables, the company's own linguistic variables are considered which are used for production terms. Raw material is one of the input in the model which is linguistic variables are "Low", "Medium" and "High". Finished goods Inventory, demand and machine breakdown inputs linguistic variables are "Low", “Medium" and "High". Due date's inputs 
linguistic variables are "Short", "Medium", and "Long". Shift Period output's linguistic variables are "Very Low", “Low", “Medium", "High" and "Very High". The constraints of the linguistic variables are shown in Table 1 and Table 2, are taken from the experts of production planning.

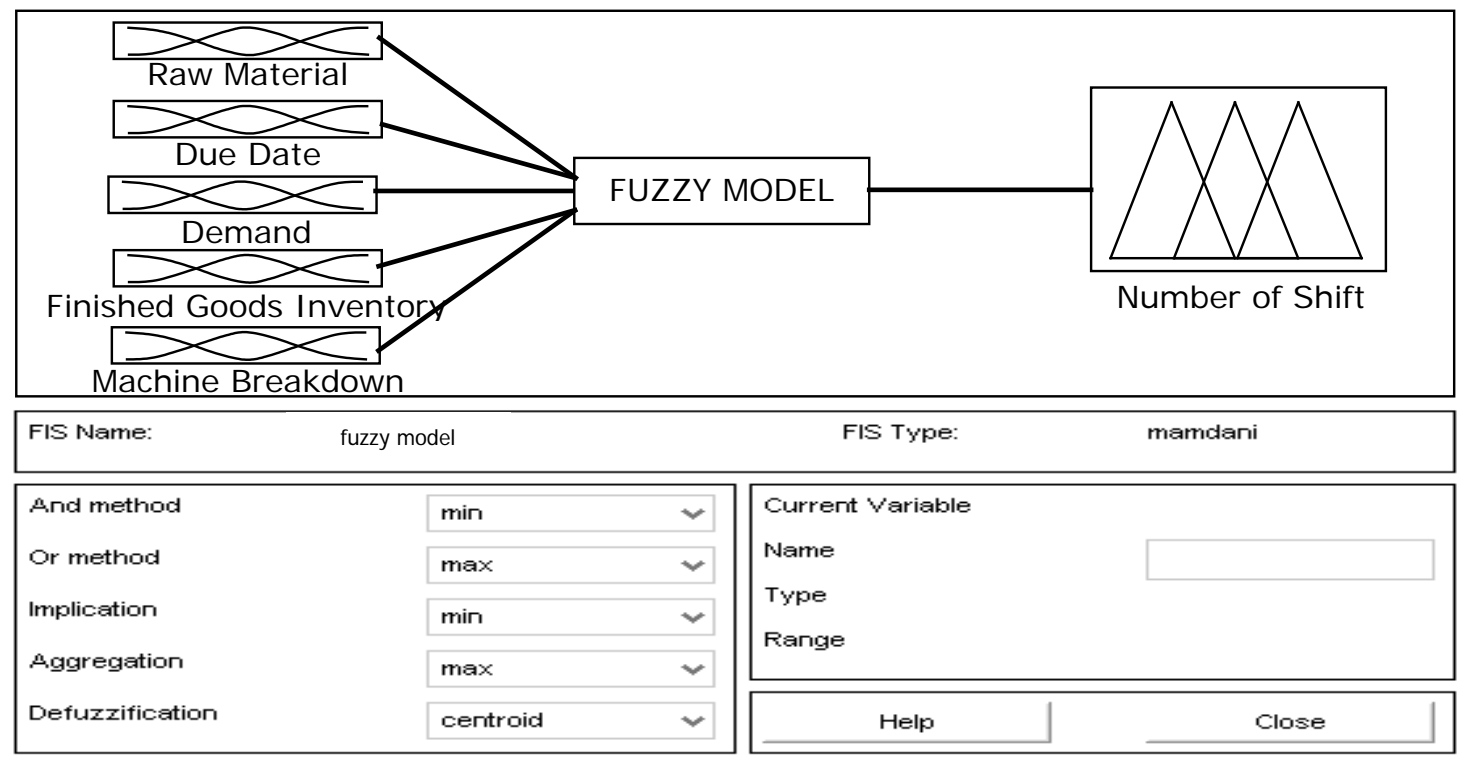

Figure 3. "FIS model for Number of Shift". Source: Own contribution

After examining the linguistic variables, membership functions are determined and entered into Matlab Fuzzy Toolbox. The Fuzzy Inference System (FIS) for input and output variables is shown in Figure 3 . In this figure, inputs variables are shown in the left side and output variable is shown in the right side.

The membership function and the linguistic variables of input and output variables are entered in Matlab Fuzzy Toolbox' Membership Function Editor. Gaussian membership function is used for each linguistic variable. Figure 4 represents the membership function and linguistic variables for one input raw material. Similarly membership function and linguistic variables are entered for other variables.

After the membership functions are determined, the rules are designated and written in Matlab Fuzzy Toolbox for evaluation. And after the needed data are entered, inputs are solved according to changing input positions for solving of fuzzified systems. With five inputs variables each having three levels, total 243 rules are developed with the help of Matlab Fuzzy Toolbox Rule Editor. Here an example of one rule is given. 
If (Raw Material is LOW) and (Due Date is LONG) and (Demand is LOW) and (Finished Goods Inventory is HIGH) and (Machine Breakdown is LOW) then (Number Of Shift is VERY LOW). Similarly 242 rules are developed and entered in the Fuzzy Toolbox Rule Editor.

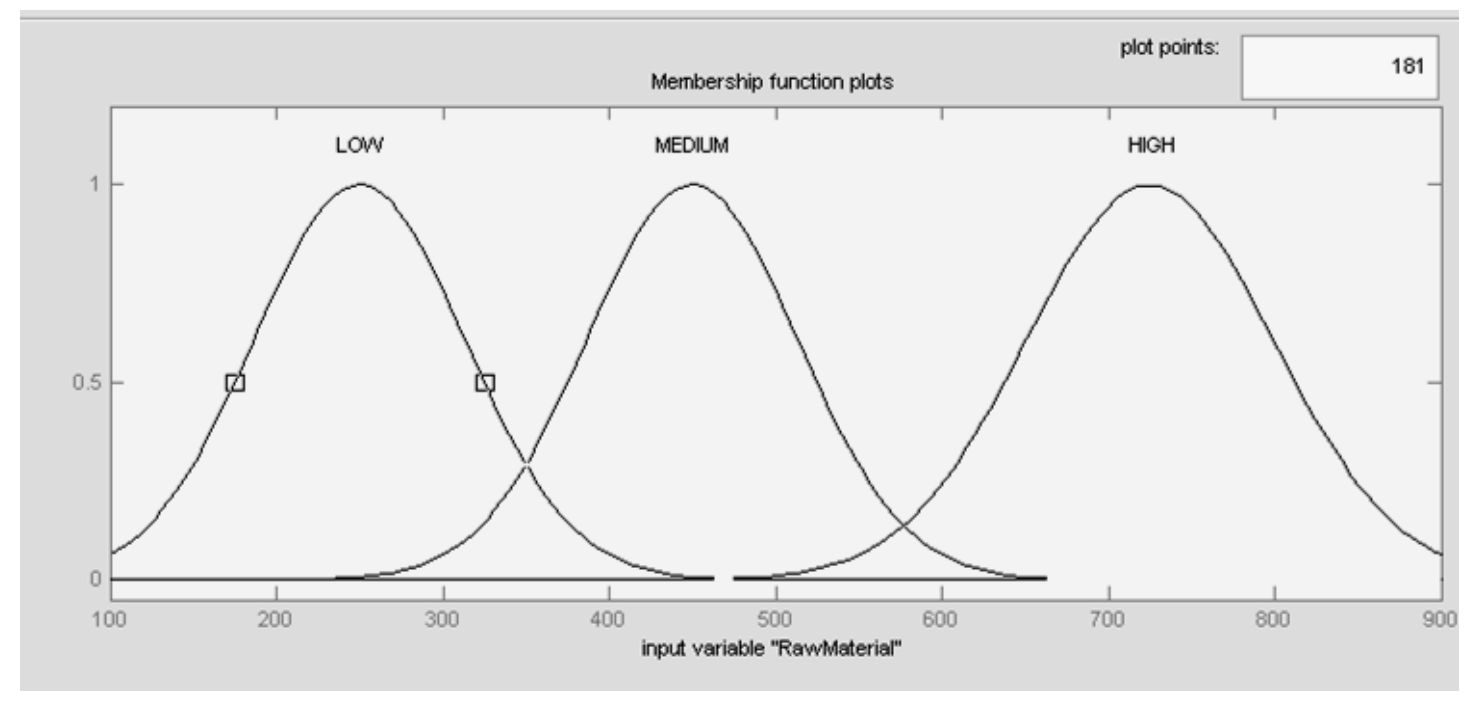

Figure 4. "FIS model for Number of Shift". Source: Own contribution

\section{Result analysis}

Data from five same kinds of manufacturing company has been considered and the result is then analyzed on the basis the data. Results from fuzzy model are compared with the company's accrued results. The data are shown in Table 3.

\begin{tabular}{|l|c|c|c|c|c|}
\hline & Raw Material & Due Date & Demand & $\begin{array}{c}\text { F/ G } \\
\text { Inventory }\end{array}$ & $\begin{array}{c}\text { Machine } \\
\text { Breakdown }\end{array}$ \\
\hline Company A & 350 & 20 & 150 & 30 & 1 \\
\hline Company B & 525 & 6 & 90 & 45 & 2 \\
\hline Company C & 225 & 15 & 60 & 70 & 1.5 \\
\hline Company D & 325 & 12 & 450 & 125 & 0.5 \\
\hline Company E & 450 & 35 & 525 & 250 & 2.5 \\
\hline
\end{tabular}

Table 3. "Data of five manufacturing companies". Source: Own contribution

Results obtained from the fuzzy model and comparison with the current result followed by company is shown is Table 4. For company A, raw material, due date, demand, finished goods inventory and machine breakdown are given inputs in the rule viewer of Matlab fuzzy logic toolbox and number of shift is shown in the rule viewer as output. The results using fuzzy logic are compared with the current 
results followed by specific company. Currently company A follows 2.5 shifts per day, but using fuzzy logic optimal number of shift is 2 . Similarly these comparisons are performed for five manufacturing companies. Optimized result is used by only Company B and Company C. No other company is using the optimal shift period. As huge cost is involved with each shift, production system should be carried with optimal number of shift.

\begin{tabular}{|c|c|c|}
\cline { 2 - 3 } \multicolumn{1}{c|}{} & $\begin{array}{c}\text { Currently company's results } \\
\text { (Number of Shift per day) }\end{array}$ & $\begin{array}{c}\text { Fuzzy Results( Number } \\
\text { of Shift per day) }\end{array}$ \\
\hline Company A & 2.5 & $1.68 \approx 2$ \\
\hline Company B & 2 & $1.61 \approx 2$ \\
\hline Company C & 2 & $1.63 \approx 2$ \\
\hline Company D & 3 & $2.25 \approx 2.5$ \\
\hline Company E & 2.5 & $1.96 \approx 2$ \\
\hline
\end{tabular}

Table 4. "Comparison of results (five companies)". Source: Own contribution

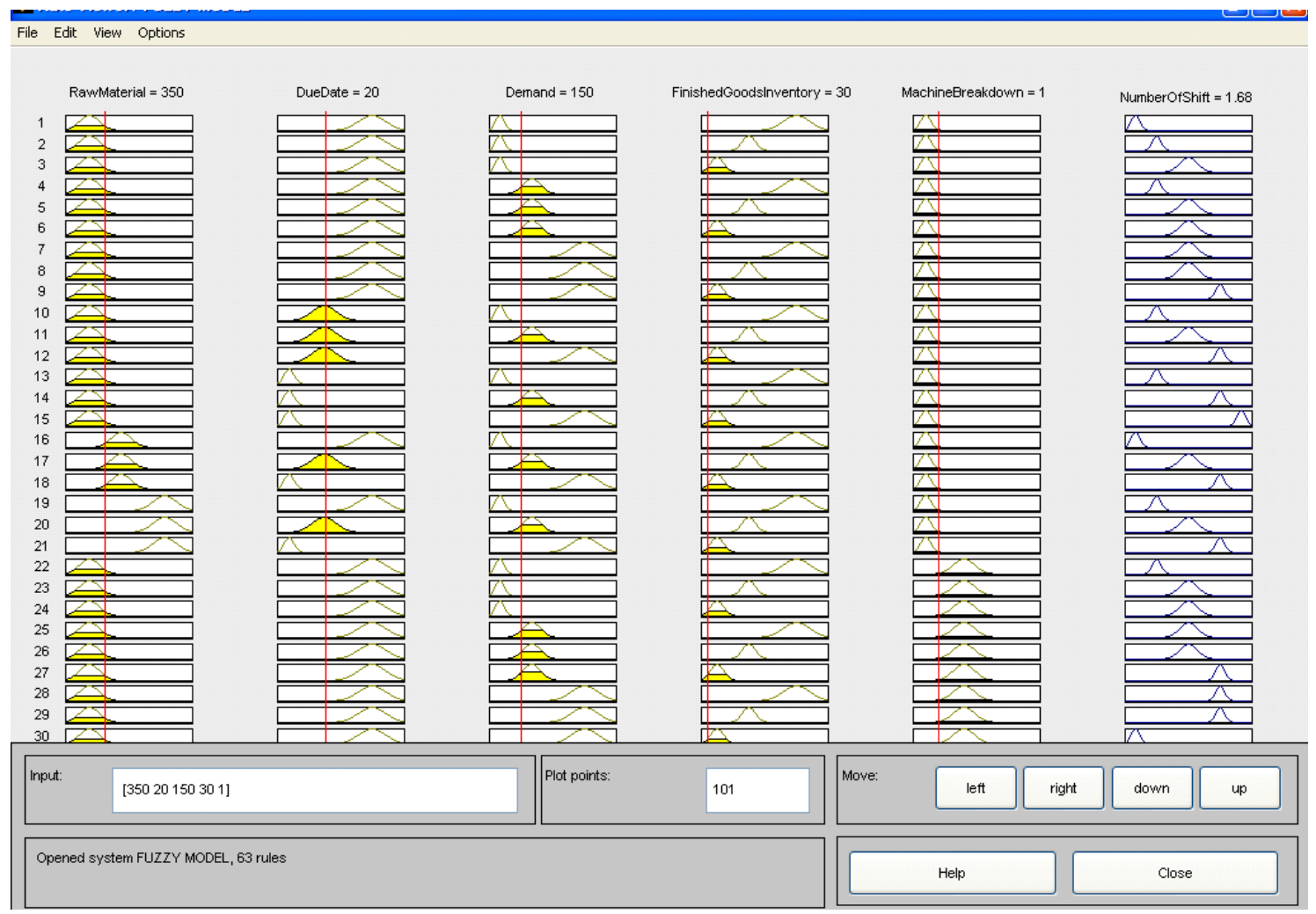

Figure 5. "View of Matlab Fuzzy Toolbox: Rule Viewer for company A". Source: Own contribution 


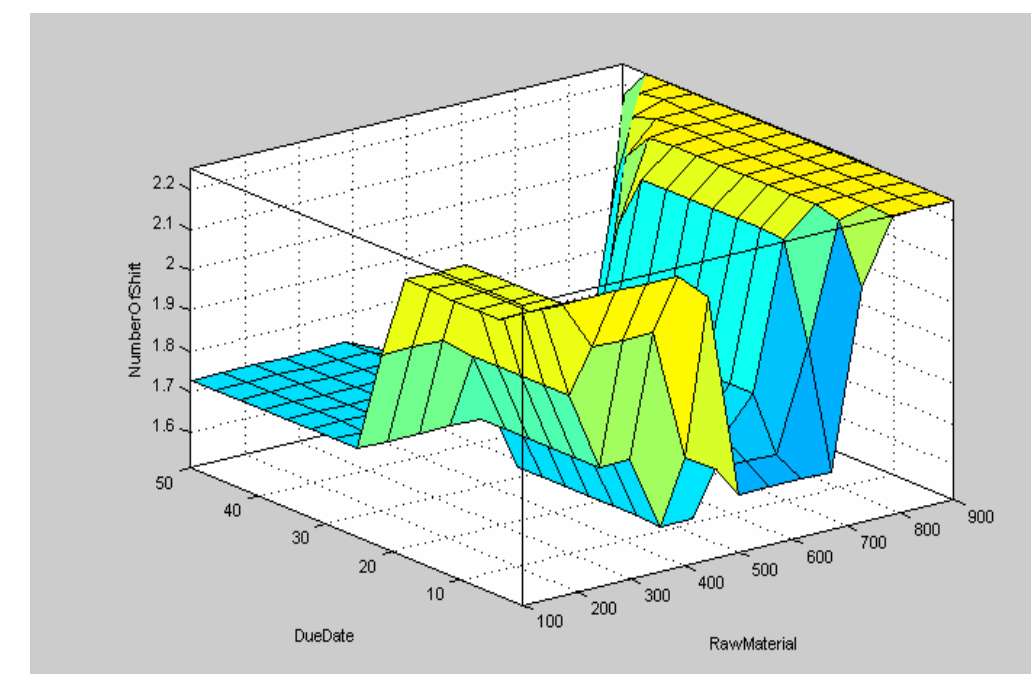

Figure 6. "Surface Analysis between Raw Material and Due Date with Number of Shift". Source: Own contribution

Rule viewer for company $A$ is represented in Figure 5. In this rule viewer inputs are given for company $A$ and optimal number of shift is automatically generated by using data of input variables and developed rules. Here inputs are raw material (350), due date (20), demand (150), finished goods inventory (30) and machine breakdown (1).

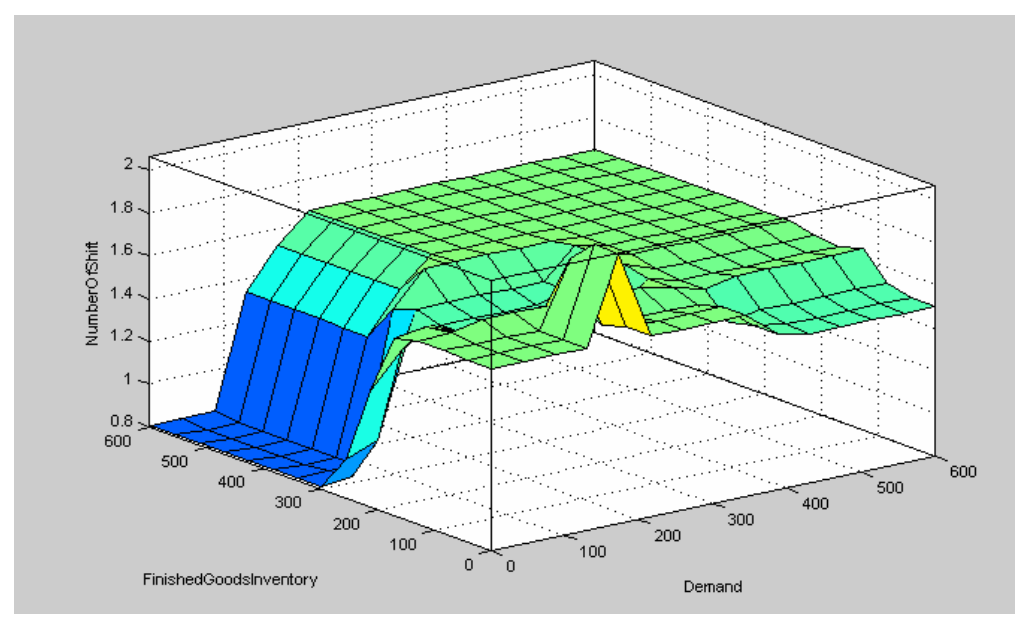

Figure 7. "Surface Analysis between Demand and F/G Inventory with Number of Shift". Source: Own contribution

The output variable number of shift is obtained 1.68 which is equivalent to 2 . This result is the optimal number of shift for Company $A$. 
Some other results which have been obtained from Matlab Fuzzy Toolbox are shown below in Figures 6, 7, 8 and 9. These figures show the effects of the inputs to the output. Figure 6 shows the how number of shift is varied with the input variables raw material and due date. Figure 7 represents the how Number of shift is varied with the input variables demand and finished goods inventory.

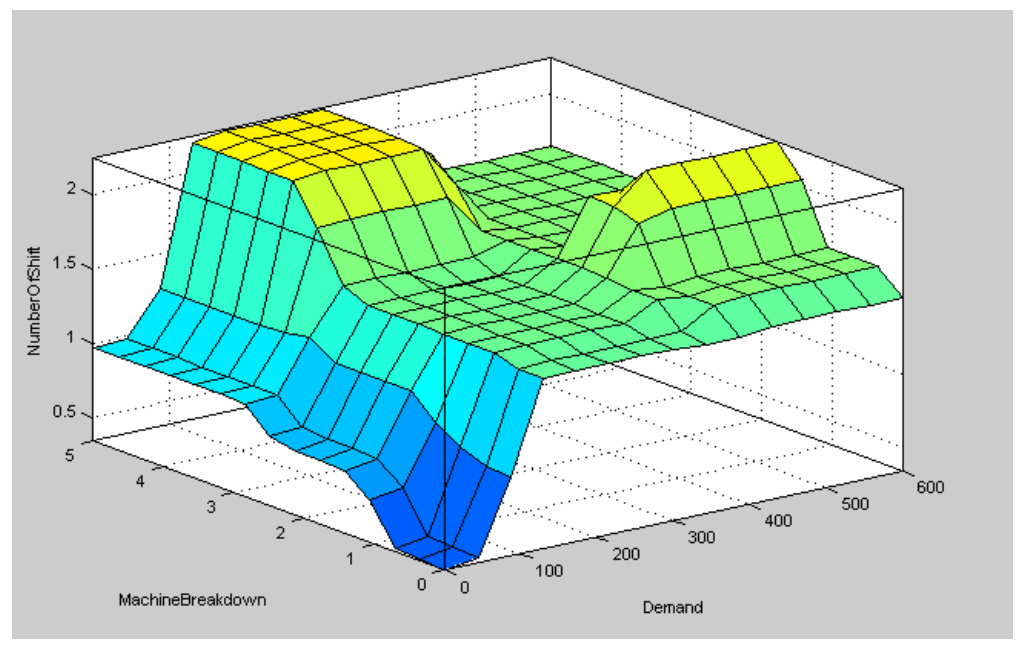

Figure 8. "Surface Analysis between Demand and Machine Breakdown with Number of Shift". Source: Own contribution

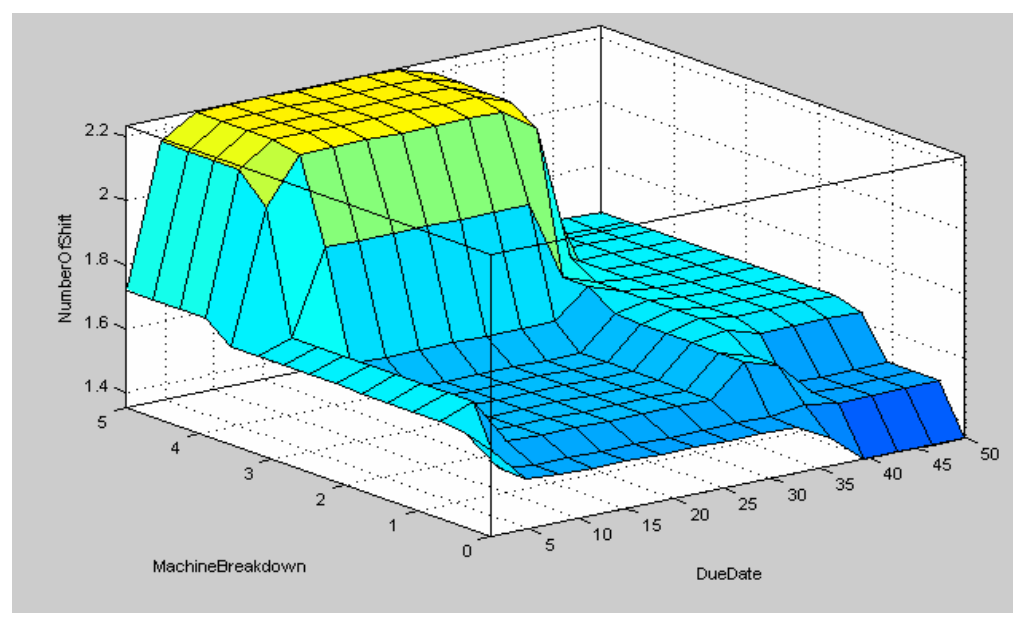

Figure 9. "Surface Analysis between Due Date and Machine Breakdown with Number of Shift". Source: Own contribution

Figure 8 shows how the number of shift is varied with the input variables demand and machine breakdown; whereas Figure 9 depicts the variation of number of shift varies with the input variables due date and machine breakdown. This variation of 
Number of shift against the input variables depends on the proposed rules as discussed earlier.

\section{Conclusions}

The number of shift is obtained by considering inventory information, customer requirements and machine reliability using the fuzzy logic is important as it gives the optimal result. Many manufacturing companies do not use optimized result which increase the total cost and decrease the total profit. So it is very necessary to know the optimal number of shift of the production system at planning stage. Fuzzy logic helps to find these optimal results if the company's forecasting data are available. In this research, using historical and forecasted data of some manufacturing companies the final result is obtained by which optimal shift period is determined. Here, five input variables (raw material, due date, demand, finished goods inventory and machine breakdown) are considered to optimize the number of shift. Some other input variables may be considered to find the result which depends on type of the production system of company. For each input and output variable Gaussian membership functions are considered to design the model. Other membership functions also may be considered to design the model. Based on the optimization of number of shifts, more effective scheduling can be performed including related variables. Raw material and finished goods inventory can also be optimized in similar way.

\section{References}

Allet, S. (2003). Handling flexibility in a generalized job shop with a fuzzy approach. European J ournal of Operations Research, 147, 312-333. doi: 10.1016/S0377-2217(02)00563-5

Axsater, S. (1990). Simple solution for a class of two-echelon inventory problems, Operations Research, 38(1), 64-69.

doi: 10.1016/S0377-2217(02)00563-5

Balan, S., Vrat, P., \& Kumar, P. (2007). Reducing the bullwhip effect in a supply chain with fuzzy logic approach. International Journal of Integrated Supply Management, 3(3), 261-282. doi:10.1504/IJISM.2007.012630 
Caglar, D. (2001). A multi-echelon spare parts inventory system with emergency lateral shipments subject to a response time constraint, Ph.D. Dissertation Thesis. Department of Industrial Engineering and Management Science, Northwestern University.

Cohen, M. A., Kleindorfer, P. R., Lee, H., \& Lee, K. (1986). Optimal stocking policies for low usage items in multi-echelon inventory systems. Naval Research Logistics Quarterly, 33, 17-38. doi: 10.1002/nav.3800330103

Deng, Y. W., Gong, J. K., Yang, J., \& Tan, L. G. (2008). An investigation on energy regeneration in a CFA6470 parallel hybrid electric vehicle using fuzzy logic. International Journal of Electric and Hybrid Vehicles, 1(3), 238-247.

doi: 10.1504/1]EHV.2008.019899

Frantti, T., \& Mahonen, P. (2001). Fuzzy logic-based forecasting model. Engineering Applications of Artificial Intelligence, 14, 189-201.

doi: 10.1016/S0952-1976(00)00076-2

Gottwald, S. (2005). Mathematical fuzzy logic as a tool for the treatment of vague information. Information Sciences, 172, 41-71. doi: 10.1016/j.ins.2005.02.004

Grabot, B. \& Geneste, L. (1994). Dispatching rules in scheduling: a fuzzy approach. International Journal of Production Research, 32(4), 903-915. doi: $10.1080 / 00207549408956978$

Graves, S. C. (1985). A multi-echelon model for repairable item with one-for-one replenishment. Management Science, 31, 1247-1256.

doi: $10.1287 / \mathrm{mnsc} 31.10 .1247$

Hedjar, R. (2008). Fuzzy control of periodic-review state-dependent production systems with unknown deterioration rate. International Journal of Operational Research, 3(6), 632-642. doi: 10.1504/IJOR.2008.019730

Hopp, W. J., Zhang, R., \& Spearman, M. L. (1999). An easily implementable hierarchical heuristic for a two-echelon spare parts distribution system. IIE Transactions, 31, 977-988. doi: 10.1080/07408179908969898 
Mahata, G. C., \& Goswami, A. (2009). A fuzzy replenishment policy for deteriorating items with ramp type demand rate under inflation. International J ournal of Operational Research, 5(3), 328-348.

doi: $10.1504 / 1 /$ OR. 2009.025200

McCahonE, C. S., \& Lee, S. (1992). Fuzzy job scheduling for a flow shop. European J ournal of Operational Research, 62, 294-301.

doi: 10.1016/0377-2217(92)90119-T

Novák, V., Perfilieva, I., \& Mockor, J. (1999). Mathematical principles of fuzzy logic. The Springer International Series in Engineering and Computer Science, $1^{\text {st }}$ edition.

Ordoobadi, S. M., \& Mulvaney, N. J. (2001). Development of a justification tool for advanced manufacturing technologies: system-wide benefits value analysis. J ournal of Engineering and Technology Management, 18, 157-184.

doi: 10.1016/S0923-4748(01)00033-9

Petroni, A., \& Rizzi, A. (2002). A fuzzy logic based methodology to rank shop floor dispatching rules. International Journal of Production Economics, 76, 99-108. doi: 10.1016/S0925-5273(01)00171-2

Ross, T. J. (1995). Fuzzy logic with engineering applications. New York: McGrawHill Inc.

Serra, G. L. O., \& Bottura, C. P. (2006). Methodology for fuzzy identification in a noisy environment. International Journal of Modellig Identification and Control, 1(4), 281-291. doi: 10.1504/1] MIC. 2006.012616

Svoronos, A., \& Zipkin, P. (1991). Evaluation of one-for-one replenishment policies for multi-echelon inventory systems. Management Science, 37(1), 68-83. doi: $10.1287 / \mathrm{mnsc} 37.1 .68$

Zadeh, L. A. (1965). Fuzzy sets. Information and Control, 8, 338-353. doi: 10.1016/S0019-9958(65)90241-X 


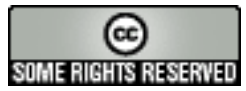

Article's contents are provided on a Attribution-Non Commercial 3.0 Creative commons license. Readers are allowed to copy, distribute and communicate article's contents, provided the author's and Journal of Industrial Engineering and Management's names are included. It must not be used for commercial purposes. To see the complete license contents, please visit http://creativecommons.org/licenses/by-nc/3.0/. 